\title{
Baudouin Dupret (dir.), La charia aujourd'hui. Usages de la référence au droit islamique
}

Paris, La Découverte, coll. « Recherches », 2012, 240 p.

\section{Belkacem Benzenine}

\section{(2) OpenEdition}

\section{Journals}

Édition électronique

URL : http://journals.openedition.org/assr/26378

DOI : $10.4000 /$ assr. 26378

ISSN : $1777-5825$

Éditeur

Éditions de l'EHESS

Édition imprimée

Date de publication : 31 décembre 2014

Pagination : 173

ISBN : 978-2-7132-2467-6

ISSN : 0335-5985

Référence électronique

Belkacem Benzenine, «Baudouin Dupret (dir.), La charia aujourd'hui. Usages de la référence au droit islamique ", Archives de sciences sociales des religions [En ligne], 168 | 2014, mis en ligne le 14 avril 2015, consulté le 22 septembre 2020. URL : http://journals.openedition.org/assr/26378; DOI : https:// doi.org/10.4000/assr.26378

Ce document a été généré automatiquement le 22 septembre 2020.

(c) Archives de sciences sociales des religions 


\section{Baudouin Dupret (dir.), La charia aujourd'hui. Usages de la référence au droit islamique}

Paris, La Découverte, coll. « Recherches », 2012, 240 p.

\section{Belkacem Benzenine}

\section{RÉFÉRENCE}

Baudouin Dupret (dir.), La charia aujourd'hui. Usages de la référence au droit islamique, Paris, La Découverte, coll. « Recherches », 2012, 240 p. 
1

La charia aujourd'hui, voilà un livre qui trouve toute sa place dans le champ des études du droit islamique. Sous la direction de Baudouin Dupret, juriste et politologue, directeur du Centre Jacques Berque à Rabat, cet ouvrage collectif apporte des éclairages à des questions primordiales sur la place de la charia et ses usages sociaux et politiques dans les mondes contemporains. Divisé en quatre parties, l'ouvrage se fait largement l'écho des débats actuels sur la référence à la normativité dans les pays islamiques et en Occident aussi. La première partie consacrée aux registres de la charia s'intéresse au cadre épistémologique qui a marqué l'évolution du droit islamique. Comment interpréter la charia selon le principe de l'ijtihad (effort individuel d'interprétation des normes juridiques)? À cette question, l'article de R. Gleave apporte des éclaircissements accompagnés de plusieurs exemples permettant au lecteur profane de mieux comprendre le fonctionnement du système juridique islamique. Sur la place de la charia, et de l'islam en général, dans les pays musulmans, N. Bernard-Maugiron montre qu'il existe différentes valeurs normatives. Qu'il y ait référence ou pas dans les Constitutions des pays musulmans, la charia est instrumentalisée dans le discours et dans les pratiques des pouvoirs politiques et des mouvements islamistes dans des questions liées à la discrimination à l'égard des femmes, l'apostasie, la censure de la création artistique, etc.

Sur les usages sociaux de la référence à la charia chez les musulmans d'Europe, F. Frégosi s'intéresse à la manière selon laquelle le discours sur la charia prend place chez les intellectuels musulmans de France comme T. Oubrou, L. Babès, A. Meddeb, T. Ramadan, lesquels s'interrogent sur la compatibilité ou non de la charia avec les législations européennes.

Quant aux usages politiques de la charia, J.-N. Ferrié, à partir du cas du Mouvement alternatif pour les libertés individuelles au Maroc (qui part de la revendication de la liberté de conscience et des réformes politiques radicales) s'intéresse au cadre institutionnel dans son lien au débat politique. L'auteur revient aussi aux déclarations de l'ex-ministre égyptien de la culture sur le voile pour montrer comment, lorsqu'il s'agit du droit islamique, une position prise par un homme politique est susceptible de créer une vive polémique.

Sur les usages de la charia dans divers pays arabes, des contributions fort intéressantes de N. Bernard-Maugiron, S. Ghamrom et J. Carlisle sur l'Égypte, le Liban, le Maroc, la Syrie et la Tunisie montrent à quel point la charia, en tant que référent s'imposant dans l'ordre juridique adopté par l'État (code de la famille, code civil et code pénal par exemple), est d'un usage problématique, à cause notamment de la diversité communautaire et confessionnelle qui caractérise les sociétés arabes. Dans le cas du 
Maroc, fondé politiquement et religieusement sur le principe de la Commanderie des croyants, M. Mouaqit montre comment la normativité de la charia "est moins déterminante dans l'ordre politique et juridique ", en expliquant son usage par la monarchie, et ceci, selon les contextes politiques (montée des courants moderniste et séculariste ou islamisme).

Dans le cas de la Tunisie où aucun texte ne fait de la charia un texte de droit, M. Ben Jémia montre comment le juge tunisien fait usage de la charia. D'après des exemples cités par l'auteur, les juges tunisiens font dans leur jurisprudence de la liberté de conscience un principe inébranlable, malgré les pesanteurs de la tradition islamique dans la sphère privée,notamment.

La troisième partie portant sur la charia au centre et à la marge est consacrée à la place du droit islamique dans des aires géographiques et culturelles différentes. Pour l'Iran, A. Amir-Arjomand, aborde la place de la charia dans l'histoire du pays, en mettant l'accent sur l'impact de la révolution de 1979 dans l'islamisation des institutions. L'auteur s'intéresse à la " modernisation » de la charia qui, s'appuyant sur des penseurs modérés comme Shabistari, montre l'intérêt de rendre la charia compatible avec les conditions des temps modernes. Sur la normativité de la charia, M. Khalil Masud aborde dans le cas du Pakistan, la diversité des discours (laïc, traditionnel, religieux, moderniste, islamiste) qui rend difficile et complexe le concept même de normativité. Cela a rendu aussi délicate la place de la charia dans l'ordre juridique et social. Dans le cas de l'Indonésie, la place de la charia pose problème dans les fondements de l'État. La contribution de A. Utrisa aborde la place de la charia dans la Constitution, la loi du mariage et les usages juridiques en Indonésie. Il retrace l'évolution du fonctionnement des tribunaux en montrant comment les juges font référence à la charia (dans le plus grand pays islamique). Le compromis semble être le modèle à suivre pour éviter les conflits confessionnels et ethniques. Sur les ambiguités de la laïcité sénégalaise, M. N’Diaye met l'accent sur la singularité du cas du Sénégal (eu égard à sa population musulmane dans sa majorité et à la marginalisation de la charia dans le code de la famille). Elle montre aussi les difficultés auxquelles sont confrontés les juges, notamment en ce qui concerne le détournement de certains codes et lois, comme c'est le cas, souvent, dans les affaires de successions.

7 Dans un autre contexte social et culturel lié à l'Amérique du Nord et à l'Europe, la quatrième partie du livre intitulée La Charia dans l'altérité est consacré aux références à la charia dans un environnement qui lui est étranger. En Belgique par exemple, la présence du référent islamique dans certains cas comme le mariage, l'héritage et l'éducation des enfants montrent, selon L.-L. Christanis, à quel point il est empêtré dans l'histoire, à cause notamment de la complexité des dimensions étatiques, socioculturelles et politiques. Dans le cas des Pays-Bas, M. Voorhoeve distingue quatre situations d'application du droit musulman. Dans un pays qui a connu ces dernières années une situation tendue à cause d'un côté de la poussée de l'extrême droite, et de l'autre de la montée du radicalisme islamique, la tolérance de l'État (que l'auteur qualifie d'historique, mais fragile) s'impose pour permettre par exemple le port du voile, et l'application de certains aspects du droit islamique à condition qu'ils ne soient pas incompatibles avec les principes fondamentaux et l'ordre public. En Allemagne, le principe de «neutralité positive » à l'égard des religions, permet, selon la contribution de M. Kütük, K.Vorholzer et J. Thielmann, une certaine forme d'organisation religieuse, sans obtenir pour autant une reconnaissance juridique formelle permettant 
aux musulmans d'avoir des droits spécifiques pour lesquels la charia serait une référence. Concernant la place de la charia dans les débats publics aux États-Unis et au Canada, Nadia Marzouki inscrit les débats dans le contexte de conditions juridiques de la liberté religieuse dans les deux pays. Il résulte selon l'auteur une différence frappante qui distingue l'attitude des "professionnels du droit» et celle de l'opinion publique et des associations de la défense des droits des minorités, ce qui rend le débat " plus conservateur et rigide".

8 Dans la conclusion de ce livre, J.-P. Bras parle des «métamorphoses de la charia » en soulignant les contours et les fonctions, les contextes et les particularités, les usages politiques et juridiques, la complexité et les difficultés des usages et des registres normatifs liés à la charia.

9 Cet ouvrage collectif, par la diversité des contextes et des thèmes relatifs à l'usage de la charia et la qualité des contributions, contribuera à s'interroger davantage sur ce que la charia a de diversité et de variété, ouvrant ainsi de nouvelles pistes de recherche quant aux implications juridiques, politiques et anthropologiques ayant trait à l'usage de la charia. Ce qui rend le livre indispensable pour toute étude sur le droit islamique et la place que la charia y occupe. 